\title{
Critical Care Among Disadvantaged Minority Groups Made Equitable: Trends Throughout the COVID-19 Pandemic
}

\author{
Diana Cristina Lopez ${ }^{1}$. Georgina Whelan ${ }^{1}$. Lisa Kojima ${ }^{1}$. Samyukta Dore ${ }^{1}$ - Saloni Lad ${ }^{1}$. Dominique Tucker ${ }^{1}$.

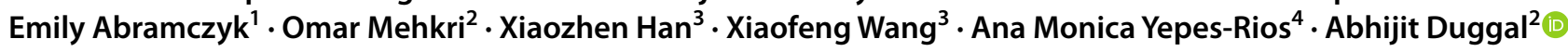

Received: 23 November 2021 / Revised: 26 January 2022 / Accepted: 27 January 2022 / Published online: 4 February 2022

(c) W. Montague Cobb-NMA Health Institute 2022

\begin{abstract}
Background US racial and ethnic minorities have well-established elevated rates of comorbidities, which, compounded with healthcare access inequity, often lead to worse health outcomes. In the current COVID-19 pandemic, it is important to understand existing disparities in minority groups' critical care outcomes and mechanisms behind these—topics that have yet to be well-explored.

Objective Assess for disparities in racial and ethnic minority groups' COVID-19 critical care outcomes.

Design Retrospective cohort study.

Participants A total of 2125 adult patients who tested positive for COVID-19 via RT-PCR between March and December 2020 and required ICU admission at the Cleveland Clinic Hospital Systems were included.

Main Measures Primary outcomes were mortality and hospital length of stay. Cohort-wide analysis and subgroup analyses by pandemic wave were performed. Multivariable logistic regression models were built to study the associations between mortality and covariates.

Key Results While crude mortality was increased in White as compared to Black patients (37.5\% vs. 30.5\%, respectively; $p=0.002$ ), no significant differences were appraised after adjustment or across pandemic waves. Although median hospital length of stay was comparable between these groups, ICU stay was significantly different (4.4 vs. 3.4, $p=0.003$ ). Mortality and median hospital and ICU length of stay did not differ significantly between Hispanic and non-Hispanic patients. Neither race nor ethnicity was associated with mortality due to COVID-19, although APACHE score, CKD, malignant neoplasms, antibiotic use, vasopressor requirement, and age were.

Conclusions We found no significant differences in mortality or hospital length of stay between different races and ethnicities. In a pandemic-influenced critical care setting that operated outside conditions of ICU strain and implemented standardized protocol enabling equitable resource distribution, disparities in outcomes often seen among racial and ethnic minority groups were successfully mitigated.
\end{abstract}

Keywords Critical care $\cdot$ ICU strain $\cdot$ COVID-19 $\cdot$ Social disparities of health $\cdot$ Racial and ethnic inequality

Diana Cristina Lopez and Georgina Whelan Shared first authorship.

Diana Cristina Lopez and Georgina Whelan contributed equally to this work.

Abhijit Duggal

duggala2@ccf.org

1 Cleveland Clinic Lerner College of Medicine of Case Western Reserve University, Cleveland, OH, USA

2 Department of Critical Care, Respiratory Institute, Cleveland Clinic, 9500 Euclid Avenue, OH 44195 Cleveland, USA

\section{Introduction}

COVID-19 has put significant strain on healthcare systems, with a high proportion of patients admitted to the ICU requiring mechanical ventilation. Studies of past and current

3 Department of Quantitative Health Sciences, Cleveland Clinic, Cleveland, OH, USA

4 Department of Internal Medicine, Cleveland Clinic, Cleveland, OH, USA 
pandemics have shown that minority groups and individuals of low socioeconomic status are disproportionately affected due to barriers of access to prevention and care [1]. US racial and ethnic minority populations are known to have higher rates of comorbidities, which, when coupled with gaps in access to healthcare, have led to worse outcomes when compared to those of their White counterparts [2,3]. These existing health disparities are compounded by situations that increase the demand and resource depletion of a healthcare system, such as a global pandemic. Studies of past pandemics, such as the 2008 H1N1 Influenza, have highlighted important disparities in exposure, susceptibility to disease, and access to care [4]. These barriers include an increased risk of being exposed to communicable diseases, increased susceptibility to illness due to pre-existing medical comorbidities, and limited access to testing and care [4].

Analysis of the COVID-19 pandemic has demonstrated this alarming trend, such as within New York City and other urban centers, where the Black community and other communities of color have been particularly affected by the pandemic [5]. These risks may be further compounded by the fact that minority groups and individuals of low socioeconomic status are more likely to have decreased access to timely intervention, an inability to undergo social distancing due to work-related demands, language barriers, and larger household sizes. Altogether, these factors could hinder individuals of disadvantaged communities from seeking medical attention, testing, and treatment, contributing to poorer outcomes [6].

The effects of COVID-19 on the health outcomes of racial and ethnic minority groups are still incompletely understood, especially within the critical care setting. Long-term mitigation of these disparities will require an assessment of the differences in COVID-19 ICU outcomes between patients who identify as Black, Hispanic, and non-Hispanic White. By identifying whether race and/or ethnicity is associated with inequalities in critical care management of COVID-19 within the Northeastern Ohio community, we hope to inform guidelines for nationwide providers to help mitigate disparities among vulnerable populations in the setting of severe disease and high acuity care. Similar to other recent data, we hypothesized that patients who identify as Black or Hispanic in Cleveland, $\mathrm{OH}$, may be more susceptible to poor COVID19 outcomes following ICU admission, including increased length of stay and increased mortality rates, as compared to non-Hispanic White patients.

\section{Methods}

\section{Cleveland Clinic COVID-19 Research Registry}

This was a retrospective cohort study utilizing the Cleveland Clinic COVID-19 ICU Registry, which compiles clinical data obtained from the electronic medical record of all patients admitted to a Cleveland Clinic Healthcare System ICU for the treatment of COVID-19. The study was approved by the Cleveland Clinic Institutional Review Board \#20-404. Patients enrolled in the COVID-19 Cleveland Clinic Registry tested positive for the SARS-CoV-2 virus via RT-PCR. The registry includes data on patient demographics, comorbidities, risk factors for contracting COVID-19 and experiencing a more severe disease course, intubation status, medications used in the treatment of COVID-19, duration of follow-up, and COVID-19 outcomes, such as length of hospital and ICU stay and mortality. The severity of illness was quantified by using the APACHE score.

\section{Subjects}

A total of 2125 adults over the age of 18 years admitted to a Cleveland Clinic ICUs in Northeastern Ohio for the treatment of COVID-19 between March and December 2020 were included in this analysis (Fig. 1). Apart from age, no other exclusion criteria were applied. Three waves of COVID-19 patients were independently studied, with each wave defined as March to June, July to September, and October to December, respectively. Within race, individuals self-identified as Black, White, or other, which includes Asian, Native Hawaiian, Pacific Islander, American Indian, Alaskan Native, or multiracial. Patients were also identified as being of either Hispanic or non-Hispanic ethnicity.

\section{Primary and Secondary Outcomes}

Our primary outcome was incidence of mortality, either during hospital admission or within the 90-day follow-up period thereafter. Our secondary outcomes were length of hospital and ICU stay.

\section{Data Analysis}

An overall analysis was performed on the entire cohort in addition to subgroup analyses for each pandemic wave, as defined above. Descriptive statistics were used to characterize the patient cohort. Patient information was described using median (25th-75th percentile) for all continuous variables and counts and percentages for all categorical variables. Wilcoxon rank sum test or Kruskal-Wallis test was used to compare differences between groups for continuous variables, and Fisher's exact test or chi-square test was applied for categorical variables. Multivariable logistic regression models were built to study the associations between the mortality outcome and pre-specified variables. Variables included in the regression model were comorbidities that were hypothesized to affect disease severity and factors important to ICU 
and COVID-19 care. In the case of missing values, only complete cases were analyzed. The analyses were two-tailed and were performed at a significance level of 0.05 . SAS 9.4 software (SAS Institute, Cary, NC) was used for all analyses. For analyses based on race, a Bonferroni correction was used for multiple comparisons, with a significance level of $0.05 / 3=0.017$ being considered significant.

\section{Results}

\section{Characteristics of COVID-19-positive patients based on race}

A total of 2125 patients tested positive for COVID-19 between March and December 2020 and required an intensive care unit (ICU) admission during their hospital course. Among the 2108 patients included in the race analysis
(17 were excluded for missing data) (Fig. 1), $41.8 \%$ were female, $33 \%$ identified as Black, and $6.7 \%$ identified as being of non-White or non-Black race (Table 1). The Black cohort was younger than the White cohort (66 vs. 71 years, $p<0.001)$. Black patients had a significantly higher prevalence of asthma (26.3\% vs. $20.4 \%, p=0.003$ ), CKD ( $48.6 \%$ vs. $36.7 \%, p<0.001)$, and diabetes $(68.1 \%$ vs. $56.0 \%, p<0.001)$ as compared to White patients. Additionally, Black patients had a lower prevalence of malignant neoplasms ( 37.3 vs. $45.0 \%, p=0.001)$. There were no significant differences between Black and White patients for chronic cardiac disease, COPD, CAD, dementia, HTN, hematological malignancies, solid organ and bone marrow transplants, malnutrition, liver disease, and immunodeficiencies. Median APACHE score, a predictor of ICU mortality, was 54 and 55 for Black and White patients, respectively $(p=0.40)$. For comparisons between Black, White, and other populations, see Supplementary Table 1.
Table 1 Patient baseline characteristics based on race (White vs. Black)

\begin{tabular}{|c|c|c|c|c|}
\hline Variable & Overall $(N=1,967)$ & Black $(N=699)$ & White $(N=1,268)$ & $p$ value \\
\hline \multicolumn{5}{|l|}{ Demographics } \\
\hline Age & $69.0[59.0,78.0]$ & $66.0[55.0,75.0]$ & $71.0[61.0,79.0]$ & $<0.001^{\mathrm{b}}$ \\
\hline Female & $830(42.2)$ & $315(45.1)$ & $515(40.6)$ & $0.056^{\mathrm{c}}$ \\
\hline \multicolumn{5}{|l|}{ Comorbidities } \\
\hline COPD & $712(36.2)$ & 251(35.9) & 461(36.4) & $0.84^{\mathrm{c}}$ \\
\hline Asthma & $443(22.5)$ & $184(26.3)$ & $259(20.4)$ & $\mathbf{0 . 0 0 3}^{\mathrm{c}}$ \\
\hline Diabetes & $1,186(60.3)$ & $476(68.1)$ & $710(56.0)$ & $<0.001^{\mathrm{C}}$ \\
\hline HTN & $1,772(90.1)$ & $644(92.1)$ & $1,128(89.0)$ & $0.024^{\mathrm{c}}$ \\
\hline CAD & $408(20.7)$ & $149(21.3)$ & $259(20.4)$ & $0.64^{\mathrm{c}}$ \\
\hline Chronic cardiac disease & $1,393(70.8)$ & $490(70.1)$ & $903(71.2)$ & $0.60^{\mathbf{c}}$ \\
\hline CKD & $805(40.9)$ & $340(48.6)$ & $465(36.7)$ & $<0.001^{\mathrm{c}}$ \\
\hline Liver disease & 431(21.9) & $165(23.6)$ & $266(21.0)$ & $0.18^{\mathrm{c}}$ \\
\hline Malignant neoplasm & $831(42.2)$ & $261(37.3)$ & $570(45.0)$ & $0.001^{\mathrm{c}}$ \\
\hline Immunodeficiency & $27(1.4)$ & $7(1.0)$ & $20(1.6)$ & $0.29^{\mathbf{c}}$ \\
\hline AIDS (HIV) & $20(1.0)$ & $10(1.4)$ & $10(0.79)$ & $0.17^{\mathbf{c}}$ \\
\hline $\begin{array}{l}\text { Solid organ or bone mar- } \\
\text { row transplant }\end{array}$ & $106(5.4)$ & $38(5.4)$ & $68(5.4)$ & $0.94^{\mathrm{c}}$ \\
\hline Malnutrition & $1,336(67.9)$ & $468(67.0)$ & $868(68.5)$ & $0.49^{\mathbf{c}}$ \\
\hline Smoking & & & & $<0.001^{\mathrm{c}}$ \\
\hline Current smoker & $158(8.2)$ & $84(12.3)$ & $74(6.0)$ & \\
\hline Former smoker & $912(47.4)$ & 291(42.7) & $621(50.0)$ & \\
\hline \multicolumn{5}{|l|}{ Severity of illness } \\
\hline APACHE score & $55.0[40.0,74.0]$ & $54.0[38.0,75.0]$ & $55.0[42.0,72.0]$ & $0.40^{\mathbf{b}}$ \\
\hline Wave & & & & $\mathrm{0.0003}^{1}$ \\
\hline 1 & $415(21.1)$ & $169(24.2)$ & $246(19.4)$ & \\
\hline 2 & $374(19.0)$ & $157(22.5)$ & $217(17.1)$ & \\
\hline 3 & 1178 (59.9) & $373(53.4)$ & $805(63.5)$ & \\
\hline
\end{tabular}

* Statistics presented as mean \pm SD, median [P25, P75], median (min, max) or N (column \%)

${ }^{\dagger} p$ values: $\mathrm{a}=$ ANOVA, $\mathrm{b}=$ Kruskal-Wallis test, $\mathrm{c}=$ Pearson's chi-square test, $\mathrm{d}=$ Fisher's exact test

${ }^{\ddagger} p$ value $<0.017$ was considered significant due to Bonferroni correction 


\section{Characteristics of COVID-19-Positive Patients Based on Ethnicity}

Among the 2064 patients included in the ethnicity analysis (61 were excluded for missing data) (Fig. 1), 4.3\% of individuals identified as Hispanic (Table 2). As compared to non-Hispanic patients, Hispanic patients within this cohort had a significantly lower prevalence of chronic cardiac disease $(52.8 \%$ vs. $71.2 \%, p<0.001)$, CKD $(20.2 \%$ vs. $41.5 \%, p<0.001)$, COPD (23.6\% vs. $36.3 \%, p=0.015)$, HTN ( $80.9 \%$ vs. $89.9 \%, p=0.007)$, and malignant neoplasms (29.2\% vs. $41.8 \%, p=0.019)$. However, Hispanic patients had a significantly higher prevalence of liver disease $(32.6 \%$ vs. $22.2 \%, p=0.022)$. No significant differences were observed in the prevalence of asthma, CAD, diabetes, solid organ and BM transplant, malnutrition, $\mathrm{HIV}$, and immunodeficiency disorders between groups. The mean APACHE score was significantly lower for Hispanic patients (49 vs. $55, p=0.037$ ), suggesting that the predicted ICU mortality at admission was higher for non-Hispanic patients within this cohort.

\section{Interventions Required During ICU Admission by Race and Ethnicity}

A similar percentage of patients within the Black and White groups required intubation during their ICU course $(41.9 \%$ vs. $42.7 \%$, respectively, $p>0.05$ ); among those intubated, the mean intubation times were comparable (7.0 vs. 8.2 days) (Table 3 ). Additionally, vasopressor requirements in the ICU were not significantly different between Black and White patients $(51.2 \%$ vs. $53.5 \%$ respectively, $p>0.05$ ). Dexamethasone, which has been studied as a potential therapeutic for COVID-19, was prescribed significantly more frequently in White than Black patients (69.6\% vs. $64.2 \%, p=0.014)$. Other COVID-19 therapeutics employed include lopinavir, ritonavir, remdesivir, hydroxychloroquine, and tocilizumab. A similar percentage of patients in each category received these medications, with the
Table 2 Patient baseline characteristics based on ethnicity

\begin{tabular}{|c|c|c|c|c|}
\hline Variable & Overall $(N=2,064)$ & Hispanic $(N=89)$ & Non-Hispanic $(\mathrm{N}=1,975)$ & $\mathrm{p}$-value \\
\hline \multicolumn{5}{|l|}{ Demographics } \\
\hline Age & $69.0[59.0,78.0]$ & $60.0[48.0,72.0]$ & $69.0[59.0,78.0]$ & $<\mathbf{0 . 0 0 1}^{\mathrm{b}}$ \\
\hline Female & $868(42.1)$ & $35(39.3)$ & $833(42.2)$ & $0.59^{\mathrm{c}}$ \\
\hline \multicolumn{5}{|l|}{ Comorbidities } \\
\hline COPD & $737(35.7)$ & $21(23.6)$ & $716(36.3)$ & $0.015^{c}$ \\
\hline Asthma & $470(22.8)$ & $24(27.0)$ & $446(22.6)$ & $0.33^{\mathrm{c}}$ \\
\hline Diabetes & $1,259(61.0)$ & $63(70.8)$ & $1,196(60.6)$ & $0.053^{\mathrm{c}}$ \\
\hline HTN & $1,848(89.5)$ & $72(80.9)$ & $1,776(89.9)$ & $0.007^{c}$ \\
\hline CAD & $422(20.4)$ & $12(13.5)$ & $410(20.8)$ & $0.096^{\mathbf{c}}$ \\
\hline Chronic cardiac disease & $1,453(70.4)$ & $47(52.8)$ & $1,406(71.2)$ & $<0.001^{\mathrm{c}}$ \\
\hline CKD & $838(40.6)$ & $18(20.2)$ & $820(41.5)$ & $<0.001^{\mathrm{c}}$ \\
\hline Liver disease & $467(22.6)$ & $29(32.6)$ & $438(22.2)$ & $0.022^{c}$ \\
\hline Malignant neoplasm & $851(41.2)$ & $26(29.2)$ & $825(41.8)$ & $0.019^{c}$ \\
\hline Immunodeficiency & $28(1.4)$ & $0(0.0)$ & $28(1.4)$ & $0.63^{d}$ \\
\hline AIDS (HIV) & $22(1.1)$ & $3(3.4)$ & $19(0.96)$ & $0.07^{\mathrm{d}}$ \\
\hline $\begin{array}{l}\text { Solid organ or bone } \\
\text { marrow transplant }\end{array}$ & $115(5.6)$ & $6(6.7)$ & $109(5.5)$ & $0.62^{\mathrm{c}}$ \\
\hline Malnutrition & $1,393(67.5)$ & $55(61.8)$ & $1,338(67.7)$ & $0.24^{\mathrm{c}}$ \\
\hline Smoking & & & & $0.002^{c}$ \\
\hline Current Smoker & $160(7.9)$ & $4(4.7)$ & $156(8.1)$ & \\
\hline Former smoker & $946(46.9)$ & $27(31.4)$ & $919(47.6)$ & \\
\hline \multicolumn{5}{|l|}{ Severity of illness } \\
\hline APACHE score & $54.0[39.0,73.0]$ & $49.0[35.0,69.0]$ & $55.0[39.0,74.0]$ & $0.037^{b}$ \\
\hline Wave & & & & $0.1770^{1}$ \\
\hline 1 & $436(21.1)$ & $25(28.1)$ & $411(20.8)$ & \\
\hline 2 & 405 (19.6) & $19(21.3)$ & $386(19.5)$ & \\
\hline 3 & $1223(59.3)$ & $45(50.6)$ & $1178(59.6)$ & \\
\hline
\end{tabular}

* Statistics presented as mean \pm SD, median [P25, P75], median (min, max), or N (column \%)

† $\mathrm{p}$ values: $\mathrm{a}=$ ANOVA, $\mathrm{b}=$ Kruskal-Wallis test, $\mathrm{c}=$ Pearson's chi-square test, $\mathrm{d}=$ Fisher's exact test 
Table 3 ICU therapies based on race (White vs. Black)

\begin{tabular}{lllll}
\hline & $\begin{array}{l}\text { Overall }(N=1,967) \\
\text { Statistics }\end{array}$ & $\begin{array}{l}\text { Black }(N=699) \\
\text { Statistics }\end{array}$ & $\begin{array}{l}\text { White }(N=1,268) \\
\text { Statistics }\end{array}$ & $p$ value \\
\hline Days intubated & $7.8[3.1,14.6]$ & $7.0[2.7,13.7]$ & $8.2[3.5,14.9]$ & $0.074^{\mathbf{b}}$ \\
Intubated & $834(42.4)$ & $293(41.9)$ & $541(42.7)$ & $0.7478^{\mathbf{c}}$ \\
Dexamethasone & $1,320(67.7)$ & $442(64.2)$ & $878(69.6)$ & $\mathbf{0 . 0 1 4}^{\mathbf{c}}$ \\
Methylprednisolone & $283(14.5)$ & $74(10.7)$ & $209(16.6)$ & $\mathbf{0 . 0 0 1}^{\mathbf{c}}$ \\
Prednisone & $199(10.2)$ & $64(9.3)$ & $135(10.7)$ & $0.33^{\mathbf{c}}$ \\
Epinephrine & $369(18.9)$ & $139(20.2)$ & $230(18.2)$ & $0.29^{\mathbf{c}}$ \\
Norepinephrine & $906(46.4)$ & $306(44.4)$ & $600(47.5)$ & $0.19^{\mathbf{c}}$ \\
Phenylephrine & $698(35.8)$ & $256(37.2)$ & $442(35.0)$ & $0.35^{\mathbf{c}}$ \\
Vasopressin & $11(0.56)$ & $5(0.73)$ & $6(0.48)$ & $0.53^{\mathbf{d}}$ \\
Remdesivir & $105(5.4)$ & $50(7.3)$ & $55(4.4)$ & $\mathbf{0 . 0 0 7}^{\mathbf{c}}$ \\
Hydroxychloroquine & $170(8.7)$ & $65(9.4)$ & $105(8.3)$ & $0.40^{\mathbf{c}}$ \\
Lopinavir-ritonavir & $10(0.51)$ & $3(0.44)$ & $7(0.55)$ & $>0.99^{\mathbf{d}}$ \\
Tocilizumab & $144(7.4)$ & $41(6.0)$ & $103(8.2)$ & $0.074^{\mathbf{c}}$ \\
Antibiotics & $1,760(90.2)$ & $606(88.0)$ & $1,154(91.4)$ & $\mathbf{0 . 0 1 3}^{\mathbf{c}}$ \\
Vasopressor use & $1037(52.7)$ & $358(51.2)$ & $679(53.5)$ & $0.321^{\mathbf{c}}$ \\
\hline
\end{tabular}

*Statistics presented as mean $\pm \mathrm{SD}$, median [P25, P75], median (min, max), or N (column \%)

${ }^{\dagger} p$ values: $\mathrm{a}=$ ANOVA, $\mathrm{b}=$ Kruskal-Wallis test, $\mathrm{c}=$ Pearson's chi-square test, $\mathrm{d}=$ Fisher's exact test

${ }^{\ddagger} p$ value $<0.017$ was considered significant due to Bonferroni correction exception of remdesivir, which was more frequently administered to Black as compared to White patients (7.3\% vs. $4.4 \%$, $p=0.007)$. For comparisons between Black, White, and other populations, see Supplementary Table 2.

Corresponding trends were appreciated when comparing Hispanic to non-Hispanic patients (Table 4). 37.1\% vs. 42.4\% Hispanic and non-Hispanic patients required intubation with a median time of 11 vs. 7.8 days, respectively $(p=0.27)$. Vasopressor requirements were comparable between ethnicities, although fewer Hispanic patients received dexamethasone as compared to non-Hispanic patients $(51.7 \%$ vs. $67.9 \%, p=0.001)$. More Hispanic patients were prescribed hydroxychloroquine $(14.6 \% \mathrm{vs}$. $8.5 \%, p=0.045)$ and lopinavir/ritonavir (3.4\% vs. $0.4 \%$, $p<0.001)$, although the use of remdesivir and tocilizumab was not significantly different.
Table 4 ICU therapies based on ethnicity

\begin{tabular}{lllll}
\hline & Overall $(N=2,064)$ & Hispanic $(N=89)$ & $\begin{array}{l}\text { Non-Hispanic } \\
(N=1,975)\end{array}$ & $p$ value \\
\hline Days intubated & $7.8[3.2,14.6]$ & $11.0[3.9,16.8]$ & $7.8[3.2,14.5]$ & $0.27^{\mathbf{b}}$ \\
Intubated & $870(42.2)$ & $33(37.1)$ & $837(42.4)$ & $0.3218^{\mathbf{c}}$ \\
Dexamethasone & $1,375(67.2)$ & $46(51.7)$ & $1,329(67.9)$ & $\mathbf{0 . 0 0 1}^{\mathbf{c}}$ \\
Methylprednisolone & $296(14.5)$ & $7(7.9)$ & $289(14.8)$ & $0.070^{\mathbf{c}}$ \\
Prednisone & $209(10.2)$ & $9(10.1)$ & $200(10.2)$ & $0.98^{\mathbf{c}}$ \\
Epinephrine & $383(18.7)$ & $11(12.4)$ & $372(19.0)$ & $0.12^{\mathbf{c}}$ \\
Norepinephrine & $943(46.1)$ & $37(41.6)$ & $906(46.3)$ & $0.38^{\mathbf{c}}$ \\
Phenylephrine & $725(35.4)$ & $26(29.2)$ & $699(35.7)$ & $0.21^{\mathbf{c}}$ \\
Vasopressin & $12(0.59)$ & $0(0.0)$ & $12(0.61)$ & $>0.99^{\mathbf{d}}$ \\
Remdesivir & $115(5.6)$ & $6(6.7)$ & $109(5.6)$ & $0.64^{\mathbf{c}}$ \\
Hydroxychloroquine & $179(8.7)$ & $13(14.6)$ & $166(8.5)$ & $0.045^{\mathbf{c}}$ \\
Lopinavir-ritonavir & $10(0.49)$ & $3(3.4)$ & $7(0.36)$ & $<\mathbf{0 . 0 0 1}^{\mathbf{d}}$ \\
Tocilizumab & $161(7.9)$ & $10(11.2)$ & $151(7.7)$ & $0.23^{\mathbf{c}}$ \\
Antibiotics & $1,843(90.0)$ & $81(91.0)$ & $1,762(90.0)$ & $0.75^{\mathbf{c}}$ \\
Vasopressor & $1077(52.2)$ & $42(47.2)$ & $1035(52.4)$ & $0.335^{\mathbf{c}}$ \\
\hline
\end{tabular}

${ }^{*}$ Statistics presented as mean \pm SD, median [P25, P75], median (min, max), or N (column \%)

${ }^{\dagger} p$ values: $\mathrm{a}=$ ANOVA, $\mathrm{b}=$ Kruskal-Wallis test, $\mathrm{c}=$ Pearson's chi-square test, $\mathrm{d}=$ Fisher's exact test 
Table 5 Patient outcomes based on race

\begin{tabular}{lllll}
\hline & Overall $(N=1,967)$ & Black $(N=699)$ & White $(N=1,268)$ & $p$ value \\
\hline LOS in ICU & $4.0[1.6,10.1]$ & $3.4[1.4,8.8]$ & $4.4[1.7,10.5]$ & $\mathbf{0 . 0 0 3}^{\mathbf{b}}$ \\
LOS in Hospital & $11.0[6.5,18.2]$ & $10.6[6.0,17.9]$ & $11.5[6.7,18.5]$ & $0.056^{\mathbf{b}}$ \\
Dead & $688(35.0)$ & $213(30.5)$ & $475(37.5)$ & $\mathbf{0 . 0 0 2}^{\mathbf{c}}$ \\
\hline
\end{tabular}

${ }^{*}$ Statistics presented as Mean \pm SD, Median [P25, P75], Median (min, max) or N (column \%)

${ }^{\dagger} p$ values: $\mathrm{a}=$ ANOVA, $\mathrm{b}=$ Kruskal-Wallis test, $\mathrm{c}=$ Pearson's chi-square test, $\mathrm{d}=$ Fisher's exact test

${ }^{\ddagger} p$ value $<0.017$ was considered significant due to Bonferroni correction

${ }^{\S} \mathrm{LOS}=$ length of stay
Table 6 Patient outcomes based on ethnicity

\begin{tabular}{lllll}
\hline & Overall $(N=2,064)$ & Hispanic $(N=89)$ & Non-Hispanic $(N=1,975)$ & $p$ value \\
\hline LOS in ICU & $4.0[1.6,10.1]$ & $3.9[1.4,9.9]$ & $4.0[1.6,10.1]$ & $0.72^{\mathbf{b}}$ \\
LOS in hospital & $11.0[6.5,18.3]$ & $10.8[5.9,16.3]$ & $11.0[6.5,18.4]$ & $0.25^{\mathbf{b}}$ \\
Dead & $708(34.3)$ & $24(27.0)$ & $684(34.6)$ & $0.14^{\mathbf{c}}$ \\
\hline
\end{tabular}

${ }^{*}$ Statistics presented as mean \pm SD, median [P25, P75], median (min, max), or N (column \%)

${ }^{\dagger} p$ values: $\mathrm{a}=$ ANOVA, $\mathrm{b}=$ Kruskal-Wallis test, $\mathrm{c}=$ Pearson's chi-square test, $\mathrm{d}=$ Fisher's exact test

LOS = length of stay

\section{Outcomes of COVID-19-Positive Patients Based on Race and Ethnicity}

Mortality was seen in a higher proportion of White patients as compared to Black patients admitted to the ICU (37.5\% vs. $30.5 \%$, respectively; $p=0.002$ ). The median length of hospital stay was similar between Black and White patients (10.6 vs. 11.5 days, $p=0.056$ ). However, the median length of ICU stay was statistically different between the two groups (3.4 vs. 4.4 days, $p=0.003$ ) (Table 5; Fig. 2). For comparisons between Black, White, and other populations, see Supplementary Table 3. Mortality was not significantly different between Hispanic and non-Hispanic patients $(27.0 \%$ vs. $34.6 \%, p=0.14)$. The median hospital and ICU LOS were similar between ethnicities (10.8 vs. 11.0 days in the hospital; 3.9 and 4.0 days in the ICU) (Table 6; Fig. 3).

Mortality rate across the 3 waves of the pandemic remained stable (Table 7). Table 8 demonstrates odds ratios for variables analyzed in the logistic regression model for mortality based on race with White patients serving as the reference group. Race was not found to be associated with mortality $(\mathrm{OR}$ (Black vs. White $)=0.83,95 \% \mathrm{CI}=0.65$ to 1.05). Factors that statistically affected mortality include APACHE score at ICU admission $(\mathrm{OR}=1.02 ; 95 \% \mathrm{CI}=1.01$ to 1.02$), \mathrm{CKD}(\mathrm{OR}=1.34 ; 95 \% \mathrm{CI}=1.05$ to 1.71$)$, malignant neoplasms $(\mathrm{OR}=1.28,95 \% \mathrm{CI}=1.03$ to 1.59$)$, antibiotic use $(\mathrm{OR}=1.69,95 \% \mathrm{CI}=1.04$ to 2.73$)$, vasopressor requirement $(\mathrm{OR}=3.97 ; 95 \% \mathrm{CI}=3.12$ to 5.05$)$, and age $(\mathrm{OR}=1.06,95 \%$ $\mathrm{CI}=1.04$ to 1.07 ).

Comparable trends were observed in the logistic regression model for mortality based on ethnicity (Table 9). While ethnicity was not associated with mortality $(\mathrm{OR}=1.23$,
Table 7 Mortality across the 3 waves of the pandemic

\begin{tabular}{lllll}
\hline & Alive $(N=1393)$ & Dead $(N=732)$ & Total $(N=2125)$ & $p$ value \\
\hline Wave & & & & $0.0691^{1}$ \\
1 & $296(66.7 \%)$ & $148(33.3 \%)$ & $444(20.9 \%)$ & \\
2 & $285(69.9 \%)$ & $123(30.1 \%)$ & $408(19.2 \%)$ & \\
3 & $812(63.8 \%)$ & $461(36.2 \%)$ & $1273(59.9 \%)$ & \\
\hline
\end{tabular}

* Statistics presented as N (row\%)

$95 \% \mathrm{CI}=0.70$ to 2.15$)$, APACHE score $(\mathrm{OR}=1.02,95 \%$ $\mathrm{CI}=1.01$ to 1.02$), \mathrm{CKD}(\mathrm{OR}=1.32,95 \% \mathrm{CI}=1.03$ to 1.70$)$, malignant neoplasms $(\mathrm{OR}=1.28,95 \% \mathrm{CI}=1.03$ to 1.60$)$, antibiotic use $(\mathrm{OR}=1.75,95 \% \mathrm{CI}=1.07$ to 2.86$)$, vasopressor requirement $(\mathrm{OR}=3.82,95 \% \mathrm{CI}=3.0$ to 4.86$)$, and age $(\mathrm{OR}=1.06,95 \% \mathrm{CI}=1.05$ to 1.07$)$ were associated with the primary outcome. The same analysis was performed for each of the waves during this pandemic. Race and ethnicity were not associated with mortality in any of the waves (Supplementary Table $4 a-c, 5 a-c)$.

\section{Discussion}

We found race and ethnicity were not associated with mortality in our cohort of COVID-19-positive ICU patients despite pre-ICU pathophysiologic differences and underlying comorbidities among the populations. However, underlying comorbidities including CKD and malignant neoplasms, as well as the severity of disease, as measured by the APACHE score, were independently associated with mortality. ICU care across race and ethnicity was similar 
Table 8 Logistic regression for mortality (race)

\begin{tabular}{|c|c|c|c|c|}
\hline \multicolumn{4}{|l|}{ Odds ratio estimates } & \multirow[b]{2}{*}{$p$ values } \\
\hline Variable & Point estimate & \multicolumn{2}{|c|}{$\begin{array}{l}\text { 95\% Wald } \\
\text { confi- } \\
\text { dence limits }\end{array}$} & \\
\hline Race (Black vs White) & 0.828 & 0.651 & 1.052 & 0.1230 \\
\hline Race (other vs White) & 1.062 & 0.671 & 1.683 & 0.7967 \\
\hline APACHE & 1.016 & 1.011 & 1.020 & $<.0001$ \\
\hline CKD & 1.339 & 1.048 & 1.710 & 0.0195 \\
\hline Dialysis & 0.912 & 0.664 & 1.252 & 0.5672 \\
\hline Diabetes & 0.809 & 0.644 & 1.017 & 0.0691 \\
\hline HTN & 0.834 & 0.546 & 1.272 & 0.3993 \\
\hline COPD & 1.029 & 0.815 & 1.298 & 0.8128 \\
\hline Liver disease & 1.170 & 0.905 & 1.513 & 0.2312 \\
\hline $\begin{array}{l}\text { Smoking history } \\
\text { Current smoker vs. others }\end{array}$ & 1.346 & 0.863 & 2.100 & 0.1897 \\
\hline $\begin{array}{l}\text { Smoking history } \\
\text { Former smoker vs. others }\end{array}$ & 1.150 & 0.913 & 1.449 & 0.2338 \\
\hline Malignant neoplasm & 1.279 & 1.028 & 1.593 & 0.0275 \\
\hline Stroke & 1.089 & 0.858 & 1.382 & 0.4857 \\
\hline Antibiotics & 1.685 & 1.039 & 2.734 & 0.0346 \\
\hline Methylprednisolone & 1.115 & 0.829 & 1.498 & 0.4718 \\
\hline Tocilizumab & 1.174 & 0.803 & 1.717 & 0.4087 \\
\hline Vasopressor use & 3.970 & 3.119 & 5.052 & $<.0001$ \\
\hline Age & 1.055 & 1.044 & 1.065 & $<.0001$ \\
\hline
\end{tabular}

* Statistics presented as odds ratio (OR)

${ }^{\dagger} p$ values is used to test whether the $\mathrm{OR}$ is equal to 1

${ }^{\ddagger} p$ value $<0.05$ was considered significant

regarding intubation, vasopressor use, and antiviral use, although dexamethasone use was found to be significantly greater in the White and Non-Hispanic groups. Additionally, mortality rates did not change throughout each phase of the COVID-19 pandemic and remained similar between racial and ethnic groups.

In a critical care setting free of the negative effects of ICU strain and inequitable resource distribution, which are consequences often associated with increased pandemic healthcare demand, this work demonstrates that equitable healthcare may be achieved, via a standardized approach, especially for patients of traditionally disadvantaged racial and ethnic minorities. With the COVID-19 pandemic, the nation has seen new challenges to system surge capacity, in response to which hospitals have implemented common operational and structural changes [7]. One study showed that of the 45 surveyed sites, nearly all implemented incident command activation changes, initially canceled elective procedures, $49 \%$ expanded ward capacity, $63 \%$ expanded ICU capacity, and some added providers to match elevated demand [7]. Within such a taxed environment, innovative protocols that allow for standardization of critical care are
Table 9 Logistic regression for mortality (ethnicity)

\begin{tabular}{|c|c|c|c|}
\hline \multicolumn{3}{|l|}{ Odds ratio estimates } & \multirow[b]{2}{*}{$p$ value } \\
\hline Variable & Point estimate & $\begin{array}{l}95 \% \text { Wald } \\
\text { confi- } \\
\text { dence limits }\end{array}$ & \\
\hline Ethnicity & 1.227 & $0.702 \quad 2.147$ & 0.4725 \\
\hline APACHE & 1.015 & $1.011 \quad 1.020$ & $<.0001$ \\
\hline CKD & 1.323 & $\begin{array}{ll}1.033 & 1.694\end{array}$ & 0.0266 \\
\hline Dialysis & 0.913 & $0.665 \quad 1.255$ & 0.5769 \\
\hline Diabetes & 0.795 & $0.632 \quad 1.000$ & 0.0504 \\
\hline HTN & 0.815 & $0.529 \quad 1.256$ & 0.3538 \\
\hline COPD & 1.025 & $0.811 \quad 1.296$ & 0.8361 \\
\hline Liver disease & 1.150 & $0.888 \quad 1.489$ & 0.2896 \\
\hline $\begin{array}{l}\text { Smoking history } \\
\text { Current smoker vs. } \\
\text { others }\end{array}$ & 1.298 & $0.831 \quad 2.028$ & 0.2512 \\
\hline $\begin{array}{l}\text { Smoking history } \\
\text { Former smoker vs. } \\
\text { others }\end{array}$ & 1.139 & $0.903 \quad 1.436$ & 0.2733 \\
\hline Malignant Neoplasm & 1.282 & $\begin{array}{ll}1.028 & 1.598\end{array}$ & 0.0273 \\
\hline Stroke & 1.054 & $0.829 \quad 1.340$ & 0.6660 \\
\hline Antibiotics & 1.747 & 1.0692 .855 & 0.0261 \\
\hline Methylprednisolone & 1.175 & $0.874 \quad 1.579$ & 0.2849 \\
\hline Tocilizumab & 1.172 & $0.796 \quad 1.726$ & 0.4201 \\
\hline Vasopressor use & 3.817 & $2.995 \quad 4.864$ & $<.0001$ \\
\hline Age & 1.056 & $1.046 \quad 1.067$ & $<.0001$ \\
\hline
\end{tabular}

integral to patient survival, as demonstrated by our study. We coordinated our ICUs' surge capacity response across our network, and with even resource distribution and adequate surge planning, this study demonstrated no difference in mortality or length of stay outcomes by race or ethnicity. Our institution's success was gleaned in part by employing surge capacity principles based on those published during 2014 for the care of the critically ill and injured during pandemics [8]. The WHO utilized the 2014 Ebola scare to develop a series of recommendations for disaster preparedness, which was adapted at our institution to devise what is termed an "all hazards" approach, consisting of ten general principles, to emergency management [9]. Some examples of this approach include taking action before a disaster is imminent, facilitating creative solutions, prioritizing the safety and well-being of hospital employees, emphasizing collaboration, anticipating resource needs, and planning for recovery[9]. Implementing this multifaceted plan was crucial to our hospital system's effective COVID-19 response [9]. Throughout the pandemic, it has also become increasingly clear that limiting system overload is vital to decreasing the disease mortality burden. For instance, Janke et al. 

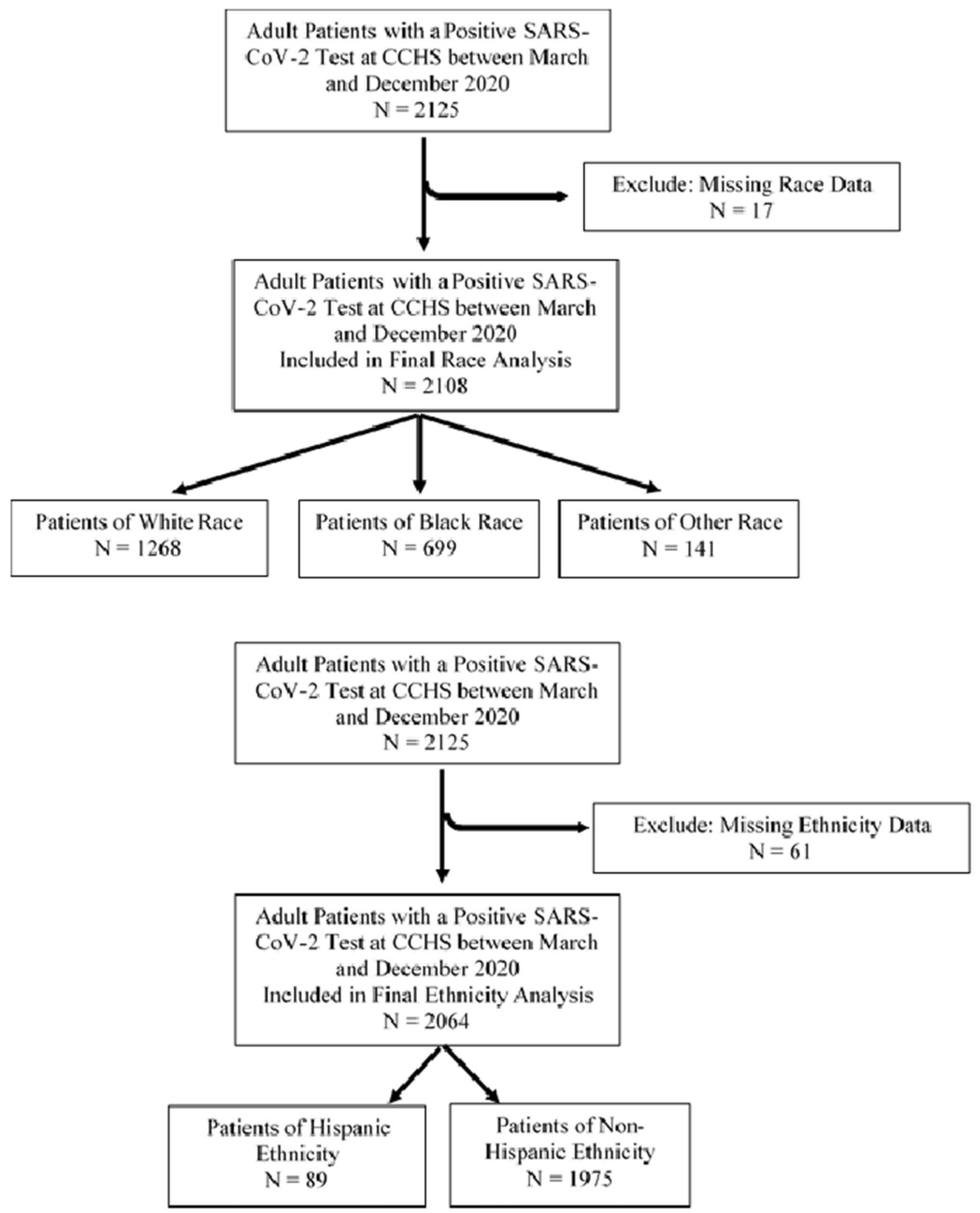

Fig. 1 Flow chart of patients included in final analysis 

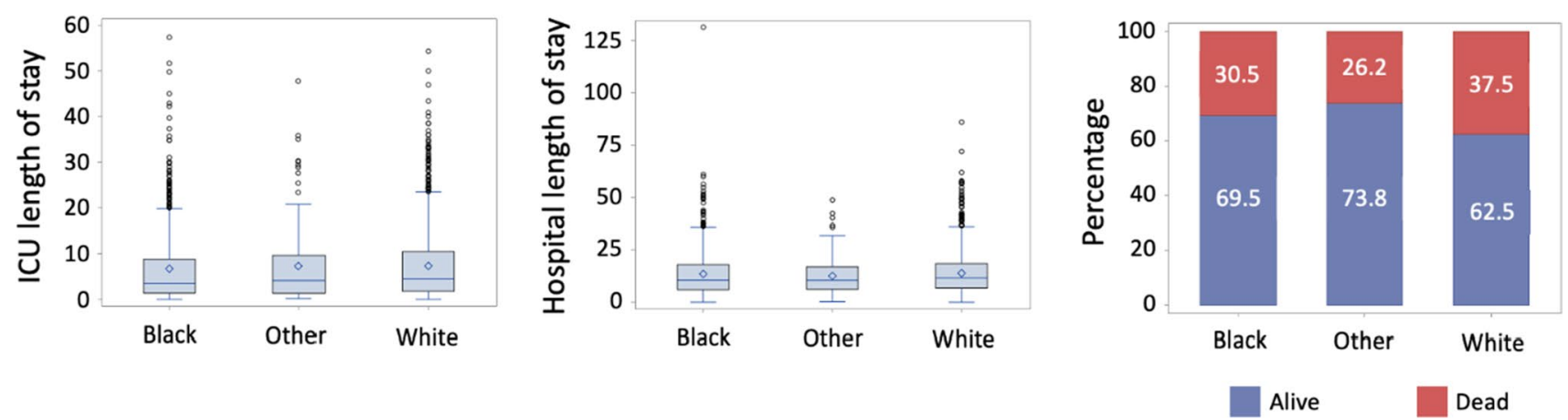

Fig. 2 Hospital LOS, ICU LOS, and Mortality based on race
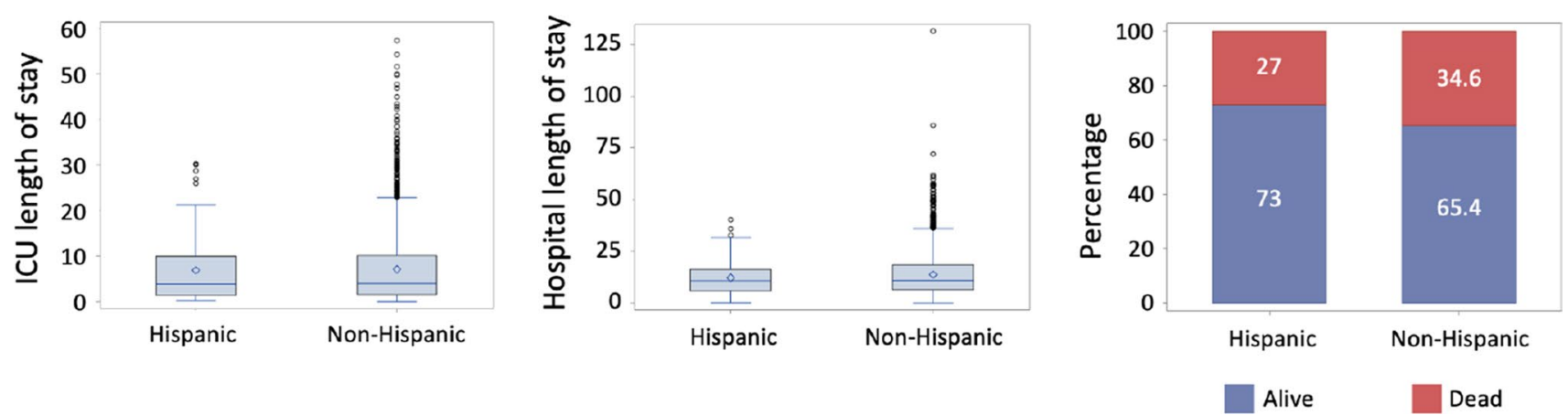

Fig. 3 Hospital LOS, ICU LOS, and Mortality based on ethnicity

demonstrated that restricted resource availability, quantified by areas with fewer ICU unit beds, nurses, and general medicine/surgical beds per COVID-19 case, was associated with statistically significant greater mortality incidence in April 2020 [10]. Similarly, Bravata et al. studied 8516 COVID-19 positive US Veterans Administration patients at 88 federal hospitals during the pandemic's height and demonstrated that ICU strain, defined by combined ICU load and demand, is associated with worse outcomes, including increased mortality [11]. They also concluded that when hospital, and specifically critical care, capacity is optimized during periods outside of acute resource strain, patients receive better quality and more standardized care, allowing for minimal opportunity for disparities between patients $[11,12]$. Such a conclusion is reassuring, as it implies that, apart from nonmodifiable variables associated with greater disease mortality, such as age and comorbidities, there are factors over which the medical community has control, including the ways in which institutional preparation and resource allocation are managed.

Early pandemic reports demonstrated a disproportionately high disease and mortality incidence among Black and Hispanic patients as compared with White patients diagnosed with COVID-19 [13]. For example, in June 2020, the CDC reported that $33 \%$ of COVID-19 cases were reported in Hispanic individuals and 22\% in Black individuals despite Hispanic and Black individuals accounting only for $18 \%$ and $13 \%$ of the US population, respectively [14]. Recently, Asch et al. showed that Black patients hospitalized with COVID19 had $11 \%$ higher odds of death, as compared to White patients even following adjustment for sociodemographic characteristics between groups. Importantly, this group found that the higher mortality among Black patients can be explained in large part by having received treatment at hospitals that care for a disproportionate number of Black patients, suggesting that hospital segregation and inequitable distribution of resources are contributing to outcome disparities [15]. On the other hand, Yehia et al. reported that hospital mortality was not higher in Black patients as compared to White patients diagnosed with COVID-19, citing mortality rates of $23.1 \%$ in hospitalized White patients and $19.2 \%$ in hospitalized Black patients within a cohort of 11,210 individuals [16], an encouraging finding dissimilar to previous literature reports but concurrent with that of this current study.

The strengths of this study are manifold. This study successfully evaluated the overall outcomes of a large number of COVID-19 patients over the course of 9 months and, moreover, more granularly analyzed this cohort by each pandemic wave to ensure no major differences in survival 
or ICU care existed among them. The patients in this study were those cared for within the Cleveland Clinic system, which includes a quaternary referral center, two tertiary centers, and six community hospitals, thus incorporating a wide variety of levels of care. Additionally, the study looked at outcomes in a critical care setting outside of that impacted by strain and limitation of resources, thus enabling a better understanding of the impact of the disease itself based on ethnicity and race.

There are several limitations to this study. First, outcomes of COVID-19 were limited to mortality and length of stay and did not investigate other lasting disease consequences such as dyspnea, cognitive changes, and symptom recovery. Second, the decreased rate of comorbidities in Hispanic as compared to non-Hispanic patients seen here, which contrasts what has traditionally been reported in the literature, suggests that this cohort may not be representative of the greater Hispanic population. Third, although the impact of racial and ethnic minority status was studied, implications of socioeconomic determinants of health and geographic factors on outcomes following critical care for COVID-19 should be further investigated in future studies.

Although we did not see a significant impact of racial and ethnic disparities on healthcare outcomes in the context of COVID-19 within Cleveland Clinic's ICUs during a circumscribed snippet in time, this should not be taken to imply that health disparities have ceased to exist. On the contrary, the conversation surrounding such pervasive inequalities and actions that should be taken to begin correcting these is precisely what we seek to incite with this work. As a tertiary and quaternary care referral center, the Cleveland Clinic's practice patterns may limit the generalizability of the findings discussed in this article. A pandemic forces hospital systems facing resource scarcity to maximize the benefits of treatment and to prioritize the allocation of resources to patients with an increased chance of survival [17, 18]. Furthermore, prior studies have shown that resource-limited hospitals and those with higher proportions of complications serve minority patients at significantly higher rates $[19,20]$. In our own study, Black and Hispanic patients possessed more comorbidities at presentation than White patients. Thus, it stands to reason that in a resource-limited health system, individuals with increased rates of comorbidities, a group likely to include those of the Black race, Hispanic ethnicity, and other minorities, may not be prioritized as large-scale decisions are made to maximize utilization of scarce resources. This strategy inevitably leads to the provision of suboptimal and inequitable care to people of color. However, armed with this knowledge, we hope that our findings serve as initial evidence that we, as healthcare providers and organizations, can begin to decrease health inequality with an intentional approach to offering uniform excellent care.
This study provides a longitudinal view of a single center's standardized critical care response to the COVID-19 pandemic. Patients, particularly those of disadvantaged racial and ethnic minority groups, are most vulnerable to disparities in care when their local healthcare systems have surpassed capacity and are increasingly resource strained. To improve upon the provision of equitable critical care within this pandemic and future scenarios of increased healthcare demand, it is important to proactively implement protocols that mitigate strain on ICUs, standardize care, and enable equitable resource distribution.

\section{Conclusion}

In this cohort of COVID-19-positive patients admitted to the ICU within the Cleveland Clinic healthcare system, neither race nor ethnicity was significantly associated with increased mortality or hospital and ICU length of stay. When not operating under critical care strain, consistent and standardized care can help translate into equitable outcomes in all individuals, including those of racial and ethnic minorities, thus benefiting traditionally disadvantaged populations.

Supplementary Information The online version contains supplementary material available at https://doi.org/10.1007/s40615-022-01254-1.

Author Contribution Daniel Moussa and Ryan Zhang contributed to the manuscript by assisting with the review of the Electronic Health Record.

Data Availability The dataset analyzed for the current study is available from the corresponding author on reasonable request.

\section{Declarations}

Ethics Approval The study was approved by the Cleveland Clinic Institutional Review Board \#20-404.

\section{References}

1. Quinn SC, Kumar S, Freimuth VS, Musa D, Casteneda-Angarita N, Kidwell K. Racial disparities in exposure, susceptibility, and access to health care in the US H1N1 influenza pandemic. Am J Public Health. 2011;101(2):285-93.

2. Iftimie S, López-Azcona AF, Vallverdú I, et al. First and second waves of coronavirus disease-19: a comparative study in hospitalized patients in Reus, Spain. PloS One. 2021;16(3):e0248029.

3. Riley WJ. Health disparities: gaps in access, quality and affordability of medical care. Trans Am Clin Climatol Assoc. 2012;123:167-72; discussion 172-174.

4. Bobo WV, Yawn BP, St Sauver JL, Grossardt BR, Boyd CM, Rocca WA. Prevalence Of combined somatic and mental health multimorbidity: patterns by Age, Sex, and race/ethnicity. J Gerontol A Biol Sci Med Sci. 2016;71(11):1483-91. 
5. Garg S, Kim L, Whitaker M, et al. Hospitalization rates and characteristics of patients hospitalized with laboratory-confirmed coronavirus disease 2019 - COVID-NET, 14 states, march 1-30, 2020. MMWR Morb Mortal Wkly Rep. 2020;69(15):458-64.

6. van Dorn A, Cooney RE, Sabin ML. COVID-19 exacerbating inequalities in the US. Lancet Lond Engl. 2020;395(10232):1243-4.

7. Mathews KS, Seitz KP, Vranas KC, et al. Variation in initial U.S hospital responses to the coronavirus disease 2019 pandemic. Crit Care Med. 2021;49(7):1038-48.

8. Hick JL, Einav S, Hanfling D, et al. Surge capacity principles: care of the critically ill and injured during pandemics and disasters: CHEST consensus statement. Chest. 2014;146(4 Suppl):e1S-e16S.

9. Orsini E, Mireles-Cabodevila E, Ashton R, Khouli H, Chaisson N. Lessons on outbreak preparedness from the Cleveland Clinic. Chest. 2020;158(5):2090-6.

10. Janke AT, Mei H, Rothenberg C, Becher RD, Lin Z, Venkatesh AK. Analysis of hospital resource availability and COVID-19 mortality across the United States. J Hosp Med. 2021;16(4):211-4.

11. Bravata DM, Perkins AJ, Myers LJ, et al. Association of intensive care unit patient load and demand with mortality rates in US Department of Veterans Affairs hospitals during the COVID-19 pandemic. JAMA Netw Open. 2021;4(1):e2034266.

12 Rubinson L. Intensive Care unit strain and mortality risk among critically Ill patients with COVID-19-there is no "me" in COVID. JAMA Netw Open. 2021;4(1):e2035041.

13. KFF. Growing data underscore that communities of color are being harder Hit by COVID-19. Available at https://www.kff. $\mathrm{org} /$ policy-watch/growing-data-underscore-communities-colorharder-hit-covid-19/. Accessed June, 20, 2021.

14. Morbidity and Mortality Weekly Report CDC. Coronavirus disease 2019 case surveillance - united states, January 22-May 30,
2020. Available at: https://www.cdc.gov/mmwr/volumes/69/wr/ mm6924e2.htm?s_cid=mm6924e2_w. Accessed June 20, 2021.

15. Asch DA, Islam MN, Sheils NE, et al. Patient and hospital factors associated with differences in mortality rates among black and white US Medicare beneficiaries hospitalized with COVID-19 infection. JAMA Netw Open. 2021;4(6):e2112842.

16. Yehia BR, Winegar A, Fogel R, et al. Association of race with mortality among patients hospitalized with coronavirus disease 2019 (COVID-19) at 92 US hospitals. JAMA Netw Open. 2020;3(8):e2018039.

17. Pandemic Influenza Plan. 2017 Update. Washington, DC: U.S. Department of Health and Human Services; 2017.

18. Biddison LD, Berkowitz KA, Courtney B, et al. Ethical considerations: care of the critically ill and injured during pandemics and disasters: CHEST consensus statement. Chest. 2014;146(4 Suppl):e145S - e155.

19. Hasnain-Wynia R, Baker DW, Nerenz D, et al. Disparities in health care are driven by where minority patients seek care: examination of the hospital quality alliance measures. Arch Intern Med. 2007;167(12):1233-9.

20. Glazer KB, Zeitlin J, Egorova NN, et al. Hospital quality of care and racial and ethnic disparities in unexpected newborn complications. Pediatrics. 2021;148(3):e2020024091.

Prior Presentations This data has not previously been presented.

Publisher's Note Springer Nature remains neutral with regard to jurisdictional claims in published maps and institutional affiliations. 